\title{
Cross-cultural adaptation, translation, and validation of the functional assessment scale for acute hamstring injuries (FASH) questionnaire for French-speaking patients
}

\author{
Médéa Locquet, Tom Willems, Clément Specque, Charlotte Beaudart, Olivier \\ Bruyère, Julien Van Beveren, Nadia Dardenne, Jean-Yves Reginster \& Jean- \\ François Kaux
}

To cite this article: Médéa Locquet, Tom Willems, Clément Specque, Charlotte Beaudart, Olivier Bruyère, Julien Van Beveren, Nadia Dardenne, Jean-Yves Reginster \& Jean-François Kaux (2020) Cross-cultural adaptation, translation, and validation of the functional assessment scale for acute hamstring injuries (FASH) questionnaire for French-speaking patients, Disability and Rehabilitation, 42:14, 2076-2082, DOI: $10.1080 / 09638288.2018 .1544669$

To link to this article: https://doi.org/10.1080/09638288.2018.1544669

View supplementary material $₫$

Published online: 22 Jan 2019.

Submit your article to this journal $₫$

III Article views: 128

Q View related articles $\sqsubset$

View Crossmark data $\nearrow$

Citing articles: 1 View citing articles ¿ð 


\title{
Cross-cultural adaptation, translation, and validation of the functional assessment scale for acute hamstring injuries (FASH) questionnaire for French-speaking patients
}

\author{
Médéa Locquet ${ }^{\mathrm{a}, \mathrm{b}}$, Tom Willems ${ }^{c}$, Clément Specque ${ }^{c}$, Charlotte Beaudart ${ }^{\mathrm{a}, \mathrm{b}}$, Olivier Bruyère ${ }^{\mathrm{a}, \mathrm{b}}$, \\ Julien Van Beveren ${ }^{\mathrm{d}}$, Nadia Dardenne ${ }^{\mathrm{e}}$, Jean-Yves Reginster ${ }^{\mathrm{a}, \mathrm{b}}$ and Jean-François Kaux ${ }^{\mathrm{c}, \mathrm{f}}$ \\ ${ }^{a}$ Department of Public Health, Epidemiology and Health Economics, University of Liège, Liège, Belgium; ${ }^{b}$ Belgium WHO Collaborating Centre \\ for Public Health Aspects of Musculoskeletal Health and Ageing, Liège, Belgium; 'Department of Sport and Rehabilitation Sciences, University \\ of Liège, Liège, Belgium; 'Haute Ecole de la Province de Liège, Liège, Belgium; ' Department of Public Health, Biostatistics, University of Liège, \\ Liège, Belgium; ${ }^{\mathrm{f}}$ Physical Medicine and Sports Traumatology Department, SportS ${ }^{2}$, FIFA Medical Centre of Excellence, University and University \\ Hospital of Liège, Liège, Belgium
}

ABSTRACT

Study design: This consisted of a translation and validation study.

Background: Acute hamstring injury is a frequent muscle strain in sports that require high explosive strength, impulsion or running phases. Therefore, the Functional Assessment Scale for Hamstring Injury questionnaire was developed to assess pain, physical activity level and ability to perform various exercises in patients with hamstring injuries. The Functional Assessment Scale for Hamstring Injury questionnaire is currently available in English, German, and Greek.

Objectives: The goal of this study was to provide a cross-culturally adapted French-translation of the FASH questionnaire and to assess its psychometric performance.

Methods: The French-translation and cross-cultural adaptation process were based on international recommendations, following six rigorous steps: (a) two initial translations from English to French; (b) synthesis of the two translations; (c) back-translations; (d) comparisons between the back-translations and the original questionnaire by an expert committee; (e) pretest; and (f) approval of the final French version of the Functional Assessment Scale for Hamstring Injury questionnaire. To validate this French version, 116 subjects (17 pathological patients, 19 patients with other muscle injury, 40 athletes at risk, and 40 healthy control athletes) were recruited to complete the Functional Assessment Scale for Hamstring Injury questionnaire. The Short Form Health Survey (SF-36) was used as a comparative questionnaire. The psychometric properties of the questionnaire were evaluated by determining the test-retest reliability after a 48-60-h interval, internal consistency, construct validity, and floor/ceiling effects.

Results: All of the items of the Functional Assessment Scale for Hamstring Injury questionnaire were translated without any major difficulties. The questionnaire showed excellent discriminative power by obtaining significantly different scores from the four groups $(p=0.01)$. Regarding psychometric performances, the test-retest reliability was excellent (IntraClass Coefficient Correlation of 0.997). Very high internal consistency was also observed (Cronbach's alpha of 0.969 ). Correlations with the physical health subscales of the SF-36 were significant and considered to be strong, indicating an excellent convergent validity. The other subscales of the SF-36 (mental health) were weakly correlated with the FASH, reflecting good divergent validity. No floor or ceiling effects were observed.

Conclusion: The French-translation of the Functional Assessment Scale for Hamstring Injury questionnaire and its cross-cultural adaptation can be considered to be successful. Functional Assessment Scale for Hamstring Injury-French questionnaire is now a reliable and valid tool for patients suffering from acute hamstring injury, and its application in clinical practice is particularly relevant.

> IMPLICATIONS FOR REHABILITATION

- The FASH-F can be considered to be discriminant, reliable and valid for the evaluation of the severity of symptoms and sports ability in individuals with hamstring injuries.

- FASH-F is now a reliable and valid tool for French-speaking patients suffering from acute hamstring injury, and its application in clinical practice is particularly relevant.

- A limitation of our study could be that the distribution between the different study groups was not homogeneous implying that our findings may not be fully representative of the general population.
ARTICLE HISTORY

Received 10 March 2018

Revised 31 October 2018

Accepted 1 November 2018

\section{KEYWORDS}

French-translation; acute injury; hamstring; FASH; psychometric properties

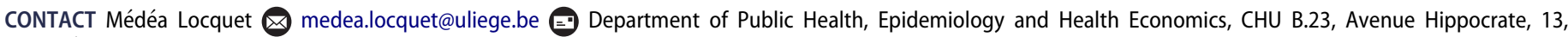
4000 Liège, Belgium.

(4) Supplemental data for this article can be accessed here.

(C) 2019 Informa UK Limited, trading as Taylor \& Francis Group 


\section{Introduction}

Hamstring muscle injuries are very common soft tissue injuries and are most frequent in soccer. Hamstring muscle injuries occur during sporting practices involving acceleration, deceleration, rapid change in direction, and jumping and frequently occur in both recreational and professional sports [1]. This lesion is noncontact-induced and appears at the posterior part of the thigh, the hamstring, a muscular complex composed of three muscles: the half-membranous, semi-tendinous and femoral biceps [2]. Growing evidence demonstrates that older age, increased quadriceps peak torque, and previous history of hamstring strain are related to an increased hamstring muscle injury risk [3].

In the field of sports sciences, the issue of hamstring muscle injuries is recognized as a public health priority. Indeed, its importance is demonstrated by its elevated prevalence, the persistence of symptoms and the long duration of recovery. Moreover, a high incidence of hamstring muscle re-injury has been established [2,4]. Given these facts, important directly and indirectly associated costs can be highlighted, such as those for treatment and clinical management, those associated with lost player payments and those related to unavailability and time lost for training and competition [5,6].

However, despite its high prevalence, hamstring muscle injury is a pathology that is still poorly understood: it is difficult to distinguish the set of factors related to the occurrence of the lesion, and it is even more complex to determine if they are a cause or a consequence. There is therefore a need to develop valid and specific measurement tools to enable a more precise assessment of not only the severity of the lesion but also its evolution until return to sports practice. There are some questionnaires dealing with hamstring injuries, but which focus on a very specific pathology of the entity [7]. Based on these statements, the Functional Assessment Scale for Acute Hamstring Injuries (FASH) was developed and validated in 2014, which measures hamstring muscle injury severity and its impact on physical function and sports ability to develop specific rehabilitation programs or to assess the treatment efficacy after hamstring injuries [8]. The FASH questionnaire consists of a set of 10 items that assess the symptoms, severity and loss of physical function in patients with hamstring muscle injury. Each item is scored on a 10-point Likert scale. The maximum possible score is 100 points, representing an individual in perfect health. It is currently the only hamstring muscle injuryspecific questionnaire and was initially developed in English [8] and Greek [8] and, after, adapted to German [9].

While French is one of the most widespread languages worldwide, the fifth most spoken language and commonly used in the sports context, no FASH adaptation for the French population is currently available. Therefore, the objectives of this study were, first, to translate the FASH questionnaire from English to French and adapt it cross-culturally according to international recommendations [10] and, second, to evaluate the psychometric performance of the questionnaire to determine its reliability and validity.

\section{Methods}

\section{French-translation of the FASH questionnaire}

The FASH questionnaire was translated according to the international recommendations for the cross-cultural adaptation of questionnaires measuring health status, implying that we followed a strict protocol composed of six steps [10]:

- Initial translation: The original questionnaire (English language) was translated twice (TF-1 and TF-2) into the target language (French) by two independent bilingual translators, one of whom is an expert in the field of health and the other is unexperienced in this area and whose mother tongue is French.

- Synthesis of the translations: During a meeting between the translators, a synthesized version (TF1-2) of the two translations (TF-1 and TF-2) was developed, taking into account any difficulties encountered when translating certain words or expressions.

- Back translation: Two new independent bilingual translators, who are experts in the field of translation and whose mother tongue is English, back translated the questionnaire to their mother tongue while blinded to the original version. From their translations, two new English versions (BT-1 and BT-2) were developed, allowing us to verify that the French translation incorporated and respected the concepts of the original version.

- Expert Committee: Essential for achieving cross-cultural equivalence of the questionnaire, the expert committee consisted of health professionals, language professionals and all four translators who were previously mentioned. A discussion between the experts took place to modify the different translations in case of linguistic inaccuracies or cultural differences. We then obtained a consensual agreement regarding the pre-final version of the questionnaire using the different translations developed previously (TF-1, TF-2, TF1-2, BT-1, and BT-2). The latter reflects a semantic, idiomatic, experiential, and conceptual equivalence. The pre-final version was proofread and corrected by a linguist, and this version was approved by the four translators to ensure the reliability, precision, and comprehensibility of the pre-final version.

- Test of the pre-final version: The pre-final version was tested on a population of 10 individuals: 5 pathological subjects (i.e., diagnosis of hamstring muscle injury) and 5 asymptomatic subjects. Each subject had to answer the questionnaire and was then asked about their ability to understand the items and answers provided, ensuring that the adapted questionnaires had the same equivalence when applied in clinical practice.

- Approval of the expert committee: The final version of the FASH-French (FASH-F) questionnaire was obtained thanks to the information obtained in the previous step and was approved by the expert committee.

\section{Validation of the FASH-F}

A validation phase was mandatory to ensure that the translation and use of the FASH-F were reliable and valid. An evaluation of its psychometric performances was therefore carried out.

\section{Recruitment of the study population}

Subjects who were at least 18 years old and provided informed consent were likely to be included in the present study except in cases of pregnancy or the presence of dorsal pain. Four different groups were established: subjects presenting a diagnosis of hamstring muscle injury (i.e., "hamstring muscle injury group"), subjects at risk of hamstring muscle injury (i.e., "At risk group"), subjects with another pathology of the lower limbs (i.e., "Other pathology group") and, finally, healthy subjects (i.e., "Control group"). The recruitment characteristics are detailed in Table 1, which also presents the specific inclusion and non-inclusion criteria for each group. The study was approved by the Ethics Committee of the University of Liège. 


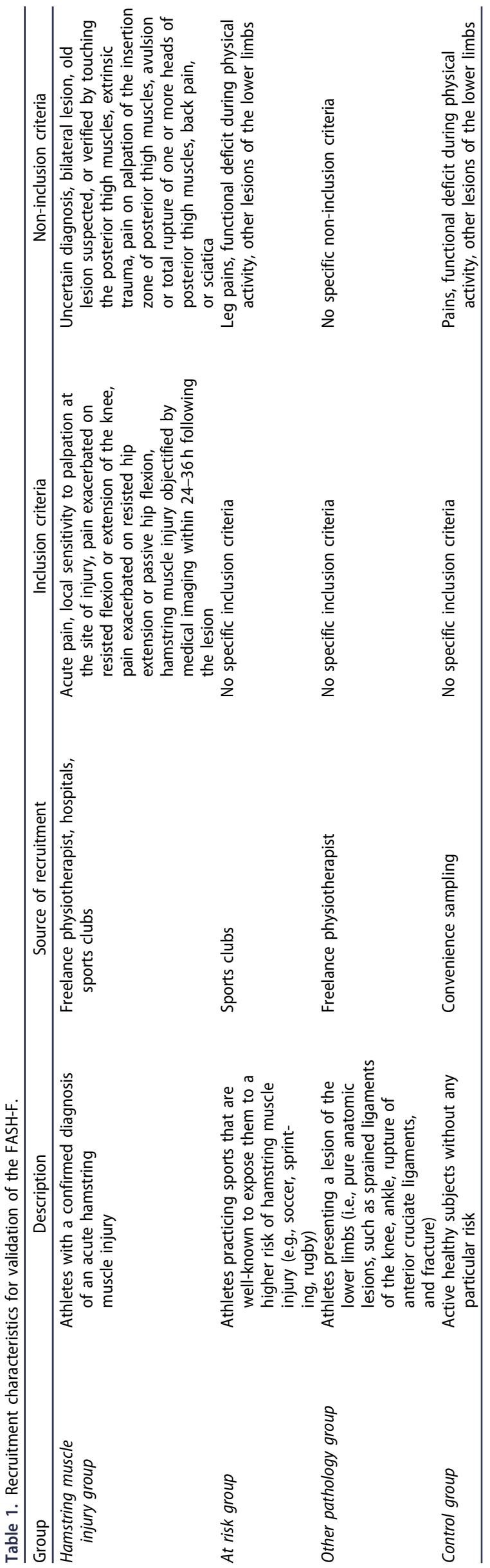

Procedure for the validation step

Once the FASH translation was carried out according to international recommendations, the translated questionnaire, the FASH-F, was submitted to all subjects between October 2016 and April 2017. Each subject completed the questionnaire twice within $48-60 \mathrm{~h}$. This choice of the test-retest interval was made to ensure that a change in clinical status had not yet occurred. Indeed, an improvement from $48 \mathrm{~h}$ post-injury can be observed [11]. A second questionnaire was also completed, the Short Form Health Survey (SF-36) [12], consisting of 36 items scored out of 100, evaluating eight concepts of physical and mental health, for an overall score of up to 100 (i.e., describing an individual in perfect health). The used of a second questionnaire made it possible to establish correlations between various items that also measure quality of life of the subjects. Because of lack of more specific lower limb questionnaire in French to compare with FASH-F, we decided to choose the SF-36 in this aim. The two questionnaires were completed by the subjects (i.e., self-administration), with an examiner present to help them in case of questions, difficulties, or for the realization of some exercises.

\section{Statistical analysis for the validation step}

Analyses were performed using SPSS Statistics 24 software (IBM, Armonk, New-York, USA). The normality of quantitative variables was tested by the Shapiro-Wilk test. According to this test, quantitative variables are expressed as the means \pm standard deviation (SD), while categorical variables are reported as the absolute and relative frequencies (\%). Results were considered statistically significant at the $5 \%$ significance level $(p<0.05)$. Furthermore, several specific statistical applications were implemented to test the psychometric performances of the FASH-F questionnaire and are described below.

Discriminative power. The discriminative ability of the FASH-F questionnaire was tested by comparing the scores (total score and individual items scores) among the four groups. Intergroup differences in regards to the clinical characteristics were tested using one-way analysis of variance (ANOVA) for continuous variables when comparing the four groups and using Student's $t$-test when comparing two groups.

Test-retest reliability. A test-retest reliability evaluation assesses the stability of the scale over time. When no health change was observed among individuals, the score of the FASH-F was expected to be unchanged. The reliability was evaluated using the Intraclass Correlation Coefficient (ICC) and 95\% confidence interval (Cl) (two-way analysis, absolute agreement), using the results obtained by the FASH-F questionnaire that was completed twice within a $48-60 \mathrm{~h}$ interval. Test-retest reliability improves as the ICC approaches 1 , and an ICC of greater than 0.7 is considered indicative of acceptable reliability [13].

Internal consistency. The estimation of homogeneity across items reflects the internal consistency of the questionnaire. The evaluation of this internal consistency was performed using Cronbach's alpha coefficient. The value of alpha varies between 0 and 1, with the internal consistency increasing as the alpha value approaches 1. A good level of internal consistency is established when the alpha value ranges from 0.70 to 0.95 [14]. Moreover, to further test this psychometric performance, we also used correlations (and $95 \% \mathrm{Cl}$ ) between the total score and each individual item. To be considered relevant (i.e., strong correlation), the correlation 
coefficient between each item and the total FASH-F score must be greater than 0.6 [15].

Construct validity. Construct validity is a psychometric property that consists of two components: convergent validity and divergent validity. Their measurement is made possible by evaluating the association, using the correlation coefficient, between the FASH-F result and subscales of the SF-36. Hypotheses were formulated concerning the two types of validity:

- Convergent validity: There is a strong correlation between the score of the FASH-F questionnaire and subscales of the SF-36 regarding the evaluation of similar concepts (i.e., "physical functioning," "role limitation due to physical problems," "bodily pain," and "general health").

- Divergent validity: There is a weak correlation between the score of the FASH-F questionnaire and subscales of the SF-36 regarding the evaluation of different concepts (i.e., "mental health," "role limitation due to emotional problem," "social functioning," and "vitality").

This requirement was considered to be fulfilled when at least $75 \%$ of the hypotheses were confirmed.

Floor and ceiling effects. Floor and ceiling effects are considered to be present if more than $15 \%$ of the population obtains a maximum score (ceiling effect) or a minimum score (floor effect). When either of these effects are present, subjects with a minimum or maximum score cannot be distinguished from each other, decreasing the discriminative power of the questionnaire.

\section{Results}

\section{French-translation of the FASH questionnaire}

Strictly following the previously detailed procedure, the FASH questionnaire was French-translated without any major difficulties. The pre-final version was tested by 10 subjects ( 5 from the pathological group and 5 from the asymptomatic group, who were subsequently integrated into the validation step), who did not reveal any problems in understanding the questionnaire or have any particular difficulty completing the questionnaire. The pre-final version thus became the definitive final version (i.e., the FASH-F questionnaire), having been validated by the expert committee (see Supporting Information Material).

\section{Validation of the FASH-F}

\section{Description of participants}

The total study sample for the FASH-F validation step consisted of 116 subjects. Of these, 17 subjects (14.6\%) presented with acute hamstring muscle injury, 19 individuals (16.4\%) presented with another lower limb pathology, and the "at risk" and "control" groups were each represented by 40 subjects (34.5\%).

The total population recruited a proportion of 88 men $(75.8 \%)$, and in each group, the proportion of men was greater than $70 \%$ (hamstring muscle injury group: $70.6 \%$, at risk group: $75.0 \%$, other pathology group: $89.5 \%$, and control group: $72.5 \%)$. The mean age was $24.2 \pm 3.6$ years in the general population and was
$24.3 \pm 2.9$ years in the hamstring muscle injury group, $23.0 \pm 2.3$ years in the group at risk, $26.4 \pm 8.9$ years in the group with other pathology and $22.9 \pm 3.0$ years in the control group. No significant differences in the mean age and sex were observed between groups (all $p$ values were $>0.05$ ). All participants were athletes, and the most represented sports were football (between $35.0 \%$ and $68.0 \%$, depending on the group), athletics (between $29.0 \%$ and $35.0 \%$, depending on the group) and rugby ( $15 \%$ for each group). Other sports, such as handball, dance, combat sports, and water sports, were also represented, but to a lesser extent. Total score to the different versions of the FASH are presented in Table 2.

\section{Discriminative power}

The total score of the FASH-F questionnaire, scored on 100 points, was $25.6 \pm 7.5$ for the pathologic group, $97.8 \pm 2.2$ for the group at risk, $80.8 \pm 8.9$ for the group of subjects presenting with another pathology, and $96.6 \pm 2.9$ for the control group. The scores differed significantly among the four groups $(F=121.7$, $p$ values $=0.01)$. When comparing the means between the hamstring muscle injury group and control group, the difference in scores was highly statistically significant ( $p$ values $<0.001$ ), and the pathological group had a significantly lower mean score. The same finding resulted when the mean scores were compared between the hamstring muscle injury group and at risk group or other pathology group (all $p$ values $<0.001$ ). The mean scores, by item, are presented in Table 3. Thus, our results showed a good capacity of the FASH-F questionnaire to discriminate individuals with and without hamstring muscle injury.

\section{Test-retest reliability}

Over a $48-60 \mathrm{~h}$ interval, all individuals responded a second time to the FASH-F questionnaire. The results demonstrated excellent test-retest reliability for the whole population using the global score (ICC: 0.997, 95\% confidence interval (Cl):0.996-0.998) (Table 4) as well as for each independent group, with an ICC varying between $0.882(95 \% \mathrm{Cl}: 0.718-0.954)$ for the other pathology group and $0.982(95 \% \mathrm{Cl}: 0.952-0.993)$ for the hamstring muscle injury group (see Table 4).

\section{Internal consistency}

A high level of internal consistency was observed, as indicated by Cronbach's alpha of the FASH-F questionnaire, which rose to 0.969 . When deleting one item at a time, reliability was considered to be unchanged, as Cronbach's alpha varied between 0.963 (item 4 deleted) and 0.973 (item 2 deleted). The correlation between the total score of the FASH-F questionnaire and each item was also assessed. The results indicated that all individual items were positively and significantly correlated with the FASH-F total score, with Spearman coefficient correlations $\left(R_{\mathrm{S}}\right)$ ranging from 0.47 (for item 6) to 0.81 (item 9) (Table 5).

\section{Construct validity}

The FASH-F total score was significantly correlated with the SF-36 subscales: strongly for subscales assessing convergent validity (all $R_{\mathrm{S}}>0.65$ except "General Health" subscale where $R_{\mathrm{S}}=0.35$ ) and

Table 2. Comparison of the total score obtained using different versions of the FASH questionnaire.

\begin{tabular}{|c|c|c|c|c|}
\hline Version of the FASH & $\begin{array}{l}\text { Hamstring muscle injury group } \\
\text { Mean } \pm \text { SD }(\mathrm{N})\end{array}$ & $\begin{array}{l}\text { At risk group } \\
\text { Mean } \pm \text { SD (N) }\end{array}$ & $\begin{array}{l}\text { Other pathology group } \\
\text { Mean } \pm S D(N)\end{array}$ & $\begin{array}{l}\text { Control group } \\
\text { Mean } \pm \text { SD (N) }\end{array}$ \\
\hline French version & $25.6 \pm 7.5(17)$ & $97.8 \pm 2.2(40)$ & $80.8 \pm 8.9(19)$ & $96.6 \pm 2.9(40)$ \\
\hline German version(9) & $42.7 \pm 29.9(16)$ & $90.1 \pm 4.7$ (19) & - & $97.5 \pm 6.3(77)$ \\
\hline English version(8) & $25.1 \pm 17.6(40)$ & $97.7 \pm 1.6(40)$ & $74.7 \pm 11.9(30)$ & $98.4 \pm 1.8(30)$ \\
\hline
\end{tabular}


Table 3. Results of the FASH-F questionnaire.

\begin{tabular}{|c|c|c|c|c|c|c|c|c|}
\hline Items & $\begin{array}{c}\text { Hamstring muscle } \\
\text { injury group } \\
n=17\end{array}$ & $\begin{array}{c}\text { At risk group } \\
n=40\end{array}$ & $\begin{array}{c}\text { Other pathology } \\
\text { group } \\
n=19\end{array}$ & $\begin{array}{l}\text { Control } \\
\text { group } \\
n=40\end{array}$ & $p$-value ${ }^{a}$ & $\begin{array}{c}p \text {-value } \\
\text { (HMl vs. at risk) }\end{array}$ & $\begin{array}{c}p \text {-value }{ }^{b} \\
\text { (HMI vs. other } \\
\text { pathology) }\end{array}$ & $\begin{array}{c}p \text {-value }^{\mathrm{b}} \\
\text { (HMl vs. control) }\end{array}$ \\
\hline Item 1 & $4.8 \pm 1.9$ & $10.0 \pm 0.0$ & $10.0 \pm 0.0$ & $10.0 \pm 0.0$ & $<0.001$ & $<0.001$ & $<0.001$ & $<0.001$ \\
\hline Item 2 & $1.2 \pm 1.9$ & $10.0 \pm 0.0$ & $2.9 \pm 2.6$ & $10.0 \pm 0.0$ & $<0.001$ & $<0.001$ & 0.03 & $<0.001$ \\
\hline Item 3 & $4.8 \pm 1.6$ & $10.0 \pm 0.0$ & $9.4 \pm 1.2$ & $9.9 \pm 0.1$ & $<0.001$ & $<0.001$ & $<0.001$ & $<0.001$ \\
\hline Item 4 & $2.0 \pm 1.3$ & $9.9 \pm 0.3$ & $8.3 \pm 2.0$ & $9.9 \pm 0.3$ & $<0.001$ & $<0.001$ & $<0.001$ & $<0.001$ \\
\hline Item 5 & $0.5 \pm 0.9$ & $9.9 \pm 0.3$ & $6.9 \pm 3.2$ & $9.8 \pm 0.5$ & $<0.001$ & $<0.001$ & $<0.001$ & $<0.001$ \\
\hline Item 6 & $3.0 \pm 1.5$ & $9.4 \pm 0.6$ & $9.8 \pm 0.9$ & $9.4 \pm 0.8$ & $<0.001$ & $<0.001$ & $<0.001$ & $<0.001$ \\
\hline Item 7 & $3.0 \pm 1.4$ & $9.5 \pm 0.6$ & $9.5 \pm 1.1$ & $9.6 \pm 0.8$ & $<0.001$ & $<0.001$ & $<0.001$ & $<0.001$ \\
\hline Item 8 & $3.6 \pm 1.9$ & $9.9 \pm 0.2$ & $9.5 \pm 1.2$ & $9.9 \pm 0.3$ & $<0.001$ & $<0.001$ & $<0.001$ & $<0.001$ \\
\hline Item 9 & $0.5 \pm 1.3$ & $9.0 \pm 1.7$ & $5.8 \pm 2.7$ & $8.0 \pm 1.9$ & $<0.001$ & $<0.001$ & $<0.001$ & $<0.001$ \\
\hline Item 10 & $2.1 \pm 2.0$ & $10.0 \pm 0.0$ & $8.6 \pm 2.9$ & $10.0 \pm 0.0$ & $<0.001$ & $<0.001$ & $<0.001$ & $<0.001$ \\
\hline Total score & $25.6 \pm 7.5$ & $97.8 \pm 2.2$ & $80.8 \pm 8.9$ & $96.6 \pm 2.9$ & 0.01 & $<0.001$ & $<0.001$ & $<0.001$ \\
\hline
\end{tabular}

a-group difference (One-way ANOVA test).

b2-group difference (Student's $t$-test).

Table 4. Test-retest reliability using ICCs for the FASH-F questionnaire.

\begin{tabular}{|c|c|c|c|c|c|}
\hline Items & $\begin{array}{l}\text { Hamstring muscle injury group } \\
\qquad n=17\end{array}$ & $\begin{array}{l}\text { At risk group } \\
\quad n=40\end{array}$ & $\begin{array}{l}\text { Other pathology group } \\
\qquad n=19\end{array}$ & $\begin{array}{l}\text { Control group } \\
\quad n=40\end{array}$ & $p$-value \\
\hline Item 1 & $0.917(0.791-0.969)$ & $1.000(1.000-1.000)$ & $1.000(1.000-1.000)$ & $1.000(1.000-1.000)$ & $<0.001$ \\
\hline Item 2 & $1.000(1.000-1.000)$ & $1.000(1.000-1.000)$ & $0.875(0.703-0.951)$ & $1.000(1.000-1.000)$ & $<0.001$ \\
\hline Item 3 & $0.931(0.823-0.974)$ & $1.000(1.000-1.000)$ & $0.836(0.621-0.935)$ & $1.000(1.000-1.000)$ & $<0.001$ \\
\hline Item 4 & $0.966(0.912-0.988)$ & $0.854(0.742-0.920)$ & $0.674(0.325-0.863)$ & $0.847(0.731-0.916)$ & $<0.001$ \\
\hline Item 5 & $1.000(1.000-1.000)$ & $0.847(0.731-0.916)$ & $0.843(0.636-0.938)$ & $0.819(0.685-0.900)$ & $<0.001$ \\
\hline Item 6 & $0.986(0.961-0.995)$ & $0.795(0.646-0.886)$ & $0.435(0.015-0.742)$ & $0.580(0.334-0.753)$ & $<0.001$ \\
\hline Item 7 & $0.973(0.929-0.990)$ & $0.854(0.742-0.920)$ & $0.960(0.898-0.985)$ & $0.716(0.525-0.839)$ & $<0.001$ \\
\hline Item 8 & $0.992(0.979-0.997)$ & $1.000(1.000-1.000)$ & $0.937(0.843-0.976)$ & $0.791(0.641-0.884)$ & $<0.001$ \\
\hline Item 9 & $1.000(1.000-1.000)$ & $1.000(1.000-1.000)$ & $0.930(0.826-0.973)$ & $1.000(1.000-1.000)$ & $<0.001$ \\
\hline Item 10 & $1.000(1.000-1.000)$ & $1.000(1.000-1.000)$ & $0.994(0.983-0.998)$ & $1.000(1.000-1.000)$ & $<0.001$ \\
\hline Total score & $0.982(0.923-0.994)$ & $0.951(0.910-0.974)$ & $0.882(0.718-0.954)$ & $0.902(0.823-0.947)$ & $<0.001$ \\
\hline
\end{tabular}

Table 5. Correlations with the total FASH-F score.

\begin{tabular}{lcc}
\hline Psychometric properties & $R_{\mathrm{S}}$ & $p$-value \\
\hline Internal consistency & & \\
Item 1 & $0.62(0.41-0.73)$ & $<0.001$ \\
Item 2 & $0.80(0.71-0.86)$ & $<0.001$ \\
Item 3 & $0.68(0.55-0.78)$ & $<0.001$ \\
Item 4 & $0.72(0.60-0.81)$ & $<0.001$ \\
Item5 & $0.77(0.67-0.84)$ & $<0.001$ \\
Item 6 & $0.47(0.29-0.62)$ & $<0.001$ \\
Item 7 & $0.61(0.46-0.73)$ & $<0.001$ \\
Item 8 & $0.64(0.49-0.75)$ & $<0.001$ \\
Item 9 & $0.81(0.72-0.87)$ & $<0.001$ \\
Item 10 & $0.67(0.53-0.77)$ & $<0.001$ \\
Convergent validity & & \\
SF-36 Physical functioning & $0.69(0.56-0.79)$ & $<0.001$ \\
SF-36 Role limitation due to physical problem & $0.67(0.53-0.77)$ & $<0.001$ \\
SF-36 Bodily pain & $0.66(0.52-0.76)$ & $<0.001$ \\
SF-36 General health & $0.35(0.15-0.52)$ & $<0.001$ \\
Divergent validity & & \\
SF-36 Mental health & $0.35(0.15-0.52)$ & $<0.001$ \\
SF-36 Role limitation due to emotional problem & $0.33(0.13-0.51)$ & $<0.001$ \\
SF-36 Social functioning & $0.35(0.15-0.52)$ & $<0.001$ \\
SF-36 Vitality & $0.37(0.17-0.54)$ & $<0.001$ \\
\hline
\end{tabular}

moderately for subscales evaluating the divergent validity $\left(R_{\mathrm{S}}\right.$ ranging between 0.33 and 0.37 ) (Table 3 ). Consequently, $87.5 \%$ of the hypotheses mentioned above were confirmed.

\section{Floor and ceiling effects}

In the pathologic group, none of the 17 subjects obtained a minimum score of 0 or a maximum score of 100 . Therefore, no floor effect or ceiling effect were observed.

\section{Discussion}

Clinical evidence shows that hamstring muscle injury is the most common muscle injury in sports. Hamstring muscle injuries have the highest rate of recurrence and are responsible for a significant amount of time spent off the field $[2,4,5]$. It is essential to have tools available to evaluate and manage this pathology for the largest possible number of individuals. Around the world, more than 274 million people can be identified as native Frenchspeaking [16], which is why it was important to develop an intercultural adaptation and validate the FASH questionnaire in French. After following rigorous translation and cross-cultural adaptation processes, the final French-version (i.e., the FASH-F questionnaire) was approved by an expert committee and, once administered, did not seem difficult for the participants to understand. Regarding validation, the psychometric properties of the questionnaire were evaluated and demonstrated the excellent performance of the questionnaire.

The total sample tested in this study consisted of 116 subjects of different ages who practiced different sports and presented with different pathologies. This diversity more accurately represents the population. The varied origins of the subjects minimized the biases induced by demographic and cultural factors. In the Greek study on the FASH questionnaire [8], the population was largely represented by field athletes and runners. The German study [9] only focused on football players. The sports most often practiced among the injured subjects of our sample (i.e., football, rugby, sprinting) were the same as those mentioned in the literature $[2,17]$. Regarding the age of the individuals in the hamstring muscle injury group, we observed that the mean age was quite young $(<25$ years), which is in agreement with the literature; the mean age at which most subjects present a hamstring muscle injury is between 17 and 22 years old [18]. The mean ages of participants in other FASH studies were similar [8,9].

Concerning the FASH questionnaire, psychometric property analyses showed that the French version was able to discriminate the pathologic group of subjects from the group at risk, group 
presenting with another pathology and control group (healthy individuals without any particular risk). Because the questionnaire was specifically developed to evaluate the severity of symptoms and sports ability in individuals with hamstring muscle injury, all of the questions included in the questionnaire are specific to individuals presenting with this type of lesion. Therefore, it is not surprising that the pathologic subjects had lower results than the non-pathologic ones. The total scores obtained during the previous studies were more or less similar to those of the FASH-F (Table 2), demonstrating a certain coherence between the versions and the reliability of our French version, even if the German hamstring muscle injury group presents higher total score, probably due to less diversity of tested participants (i.e., only football players in the German version).

The FASH-F questionnaire showed excellent test-retest reliability and therefore excellent stability over time since the ICC of the total score was 0.997 . In the case of clinical use, the ICC should be greater than 0.900 [13] to indicate a high test consistency. Our results were similar to the Greek and German studies [8,9], which also showed excellent questionnaire reliability (ICC higher than 0.900). If we evaluate the groups separately, all of the results showed excellent reliability, with an ICC ranging from 0.882 (other pathology group) to 0.982 (hamstring muscle injury group). The different items also had ICCs greater than 0.900, which demonstrated excellent reliability. Nevertheless, it appears that some answers varied more or less strongly depending on the item. Item 6 , specific to the pain felt in the hamstring, is the most demonstrative example. The ICC for he pathological groups indicated a high fidelity $(I C C=0.986)$, implying that the parameter evaluated by item 6 did not vary (or varies very little) between the first and second evaluation (48-60 h difference). This item therefore seems specific for the lesion and varies only slightly during the first few days of developing the lesion. Conversely, this item would have moderate reliability for the other pathology group $(I C C=0.435)$. The presence of another lesion seems to influence the pain felt in the hamstring. This also suggests that the specific characteristics of the questionnaire were designed for patients with hamstring muscle injury and were therefore more adapted for the pathological population.

A strong internal consistency was observed (Cronbach's alpha of 0.969 ). The items are therefore very sensitive to the concepts being evaluated. Other FASH studies also found consistent Cronbach's alphas with strong internal consistency: Cronbach's alpha between 0.917 [8] and 0.980 [9]. However, even if a result higher than 0.9 is sometimes considered desirable [19], it can also be a sign of too similar statements, paradoxically diminishing the real reliability of the scale [20].

Our study is the only study that incorporated the Spearman correlation to evaluate the correlation between items and total score of the questionnaire. All of the correlations were statistically significant. We note that all of the items, expect item 6 , had a coefficient higher than 0.6 , which is representative of a strong correlation and thus of very good internal consistency.

There was also excellent construct validity (i.e., convergent and divergent) of the FASH-F, with $87.5 \%$ of our hypotheses being confirmed. The Greek study also demonstrated good construct validity [8]. This study also determined the construct validity using the VISA-H questionnaire [7] and also focused on lesions of the hamstrings. It would have been interesting to also use this questionnaire to obtain even more robust results concerning the construct validity; however, to our knowledge, this questionnaire is not available in the French language yet.
It is also important to highlight that one limitation of our study could be that the distribution between the different study groups was not homogeneous. Some groups may, therefore, have greater statistical power. However, we separated the statistical analyses by group to observe the impact of each group in addition to the general trend. Another important limitation is the low proportion of individuals with hamstring muscle injury (i.e., 17 subjects) in our analysis. However, no guidelines currently exist regarding the ideal number of individuals to be included in a questionnaire validation study. If we refer to the other studies regarding the FASH questionnaire, our sample is similar or even superior to others (e.g., the German version is interested in 16 subjects), with standard deviations roughly in the same range. Another limitation of the present study is related to its cross-sectional design, which did not allow us to measure the sensitivity to change. This assessment is however essential in other contexts, for example, in assessing therapeutic intervention. The more sensitive a questionnaire is, the more likely it will show an effect of the intervention. In the study that validated the Greek the FASH questionnaire [8], the researchers followed a longitudinal design, which allowed them to assess a minimal clinical difference, reaching 3.05 points (the necessary value to identify a clinically relevant change of patient health status). It would have been interesting to bring additional values into our psychometric validation.

\section{Conclusion}

Given its excellent psychometric performance, the FASH-F can be considered to be discriminant, reliable and valid for the evaluation of the severity of symptoms and sports ability in individuals with hamstring muscle injury. The use of the FASH-F in French-speaking patients with acute hamstring muscle injury is now possible and fully relevant.

\section{Disclosure statement}

No potential conflict of interest was reported by the authors.

\section{References}

[1] Croisier J-L, Ganteaume S, Binet J, et al. Strength imbalances and prevention of hamstring injury in professional soccer players. Am J Sports Med. 2008;36:1469-1475.

[2] Croisier J-L. Factors associated with recurrent hamstring injuries. Sport Med. 2004;34:681-695.

[3] Freckleton G, Pizzari T. Risk factors for hamstring muscle strain injury in sport: a systematic review and metaanalysis. Br J Sport Med. 2013;47:351-358.

[4] Petersen J, Hölmich P. Evidence based prevention of hamstring injuries in sport. Br J Sport Med. 2005;39:319-323.

[5] Gabbe BJ, Finch CF, Bennell KL, et al. Risk factors for hamstring injuries in community level Australian football. Br J Sport Med. 2005;39:106-110.

[6] Gabbe BJ, Bennell KL, Finch CF, et al. Predictors of hamstring injury at the elite level of Australian football. Scand J Med Sci Sports. 2006;16:7-13.

[7] Cacchio A, De Paulis F, Maffulli N. Development and validation of a new visa questionnaire (VISA-H) for patients with proximal hamstring tendinopathy. $\mathrm{Br} J$ Sports Med. 2014;48:448-452.

[8] Malliaropoulos N, Korakakis V, Christodoulou D, et al. Development and validation of a questionnaire (FASHFunctional Assessment Scale for Acute Hamstring Injuries): 
to measure the severity and impact of symptoms on function and sports ability in patients with acute hamstring injuries. Br J Sports Med. 2014;48:1607-1612.

[9] Lohrer $H$, Nauck T, Korakakis $V$, et al. Validation of the FASH (Functional Assessment Scale for Acute Hamstring Injuries) questionnaire for German-speaking football players. J Orthop Surg Res. 2016;11:130.

[10] Beaton DE, Bombardier C, Guillemin F, et al. Guidelines for the process of cross-cultural adaptation of self-report measures. Spine (Phila Pa 1976). 2000;25:3186-3191.

[11] Malliaropoulos N, Papacostas E, Kiritsi O, et al. Posterior thigh muscle injuries in elite track and field athletes. Am J Sports Med. 2010;38:1813-1819.

[12] Brazier JE, Harper R, Jones NM, et al. Validating the SF-36 health survey questionnaire: new outcome measure for primary care. BMJ. 1992;305:160-164.

[13] Bartko JJ. The intraclass correlation coefficient as a measure of reliability. Psychol Rep. 1966;19:3-11.
[14] Tavakol M, Dennick R. Making sense of Cronbach's alpha. Int J Med Educ. 2011;2:53-55.

[15] Taylor R. Interpretation of the correlation coefficient: a basic review. J Diagn Med Sonogr. 1990;6:35-39.

[16] Estimation des francophones - Organisation internationale de la Francophonie [Internet]. 2017 [cited 2017 Dec 21]. Available from: https://www.francophonie.org/Estimationdes-francophones.html

[17] Garrett WE. Muscle strain injuries. Am J Sports Med. 1996; 24:S2-S8.

[18] Woods C, Hawkins RD, Maltby S, et al. The Football Association Medical Research Programme: an audit of injuries in professional football-analysis of hamstring injuries. Br J Sports Med. 2004;38:36-41.

[19] Bland JM, Altman DG. Cronbach's alpha. BMJ. 1997;314: 572.

[20] DeVellis RF. Scale development: theory and applications. Thousand Oaks (CA): Sage Publications; 2003. 171 p. 